\title{
$B$ Decays, the Unitarity Triangle, and the Universe
}

\author{
Adam F. Falk \\ Department of Physics and Astronomy \\ The Johns Hopkins University \\ 3400 North Charles Street \\ Baltimore, Maryland 21218 U.S.A.
}

September 5, 2018

\begin{abstract}
A review is given of recent developments in the physics of flavor. Current constraints on the Cabibbo-Kobayashi-Maskawa matrix are discussed and related to the recent measurements of $\epsilon^{\prime} / \epsilon, \sin 2 \beta$ and $K^{+} \rightarrow \pi^{+} \nu \bar{\nu}$. A brief review is given of the connection between $\mathrm{CP}$ violation in $B$ decays and electroweak baryogenesis. Finally, there is an extensive discussion of how present and proposed experiments in $K$ and $B$ physics can constrain the pattern of flavor changing processes at low energies and, one hopes, eventually provide unambiguous evidence of physics beyond the Standard Model.
\end{abstract}

Plenary talk presented at the

XIX International Symposium on Lepton and Photon Interactions at High Energies Stanford University, August 9-14, 1999 


\section{$B$ Decays, the Unitarity Triangle, and the Universe}

Adam F. Falk

Department of Physics and Astronomy

The Johns Hopkins University, Baltimore, Maryland 21218

\section{Introduction}

The past year has seen a number of exciting experimental developments which have advanced our understanding of the physics of flavor. These include the confirmation by $\mathrm{KTeV}$ [1] and NA48 [2] of the NA31 result [3] for $\operatorname{Re}\left(\epsilon^{\prime} / \epsilon\right)$ in $K_{L} \rightarrow \pi \pi$, the first measurement by $\mathrm{CDF}$ [4 of $\sin 2 \beta$ in $B \rightarrow J / \psi K_{S}$, and the observation of a single event in $K \rightarrow \pi^{+} \nu \bar{\nu}$ by BNL-AGS-E787 [5]. In addition, 1999 has seen the startup of the next generation of $e^{+} e^{-} B$ Factory experiments: BaBar at PEPII (SLAC), BELLE at KEK-B (KEK), and CLEO-III at CESR (Cornell). The first physics results from these machines are expected in 2000. The fixed target experiment HERA-B, operating in the HERA accelerator at DESY, will soon be taking physics data as well. For high statistics studies of the $K$ system, KLOE at the DA $\Phi$ NE $\phi$ Factory (Frascati) has also begun to take data this year.

Having been assigned such an ambitious title, my plan for this talk is to locate these various developments within a broader context. How do they all fit together and relate to each other? What do they signify for the next decade of particle physics? And most important, what is the role of such "low-energy" high energy physics in the anticipated era of new discoveries at the Tevatron and LHC? In short, what do we know now about the physics of flavor, and where do we hope to go in the future?

\section{The Standard Model at low energies}

We begin by recalling what the Standard Model looks like at "low" energies, by which I mean renormalization scales $\mu$ below about $10 \mathrm{GeV}$. I will refer to this theory as the Low Energy Standard Model, or LESM. At these energies we have a theory with five quarks $(u, d, s, c, b)$ and six leptons $\left(e, \mu, \tau, \nu_{e}, \nu_{\mu}, \nu_{\tau}\right)$. There is unbroken $S U(3)_{C} \times$ $U(1)_{E M}$ gauge symmetry, with eight massless gluons and a massless photon. There are Dirac masses for the quarks and charged leptons, and perhaps also Majorana masses for the neutrinos. The gauge interactions and mass terms are renormalizable

operators in the effective lagrangian. With the exception of neutrino masses, these interactions also conserve individual flavor quantum numbers. 
In the LESM, all flavor changing processes are mediated by nonrenormalizable interactions. The most important of these are four-fermion operators of mass dimension six, of the form

$$
\frac{1}{M^{2}} \bar{\psi}_{1} \Gamma \psi_{2} \bar{\psi}_{3} \Gamma \psi_{4}
$$

and fermion-gauge field interactions of dimension five, of the form

$$
\frac{1}{M} \bar{\psi}_{1} \Gamma^{\mu \nu} \psi_{2} F_{\mu \nu}
$$

Here $\psi_{i}$ are fermion fields, $\Gamma$ and $\Gamma^{\mu \nu}$ are combinations of Dirac matrices, $F_{\mu \nu}$ is a color or electromagnetic gauge field strength, and $M$ is a large mass scale. For example, neutron $\beta$ decay is mediated by the operator $\bar{u} \gamma^{\mu}\left(1-\gamma^{5}\right) d \bar{e} \gamma_{\mu}\left(1-\gamma^{5}\right) \nu_{e}$, the decay $K^{-} \rightarrow \pi^{-} \pi^{0}$ by $\bar{u} \gamma^{\mu}\left(1-\gamma^{5}\right) s \bar{d} \gamma_{\mu}\left(1-\gamma^{5}\right) u$, and $B^{0}-\bar{B}^{0}$ mixing by $\bar{b} \gamma^{\mu}\left(1-\gamma^{5}\right) d \bar{b} \gamma_{\mu}(1-$ $\left.\gamma^{5}\right) d$. In each case, the scale $M$ is $M_{W}$. There are hundreds of such operators in the LESM, responsible for an enormous variety of flavor changing interactions.

In any effective field theory, nonrenormalizable operators are generated by the exchange of heavy particles which have been "integrated out" of the theory at shorter distance scales. In the case of the LESM, some heavy particles which play this role already have been observed directly at higher energies. First, we know that there is an electroweak gauge symmetry which is realized nonlinearly, $S U(2)_{L} \times U(1)_{Y} \rightarrow$ $U(1)_{E M}$, with three group generators broken by the the vacuum expectation value $v=246 \mathrm{GeV}$. These generators correspond to the observed $W$ and $Z$ gauge bosons, with $M_{W} \simeq 80 \mathrm{GeV}$ and $M_{Z} \simeq 91 \mathrm{GeV}$. Second, the $t$ quark has been seen, with mass $m_{t} \simeq 175 \mathrm{GeV}$. Third, there are "hard" components of the $S U(3)_{C} \times U(1)_{E M}$ gauge fields, with $p^{2}>\mu^{2}$, which have also been removed from the theory and their effects absorbed into the LESM lagrangian. (Note that, except for the Goldstone bosons eaten by the $W$ and $Z$, no Higgs particle or other remnant of the electroweak symmetry breaking sector has been observed, and there is little experimental evidence, even indirect, as to its nature.)

The couplings of the $W, Z$ and $t$ are fixed by gauge symmetry, so virtual $W, Z$ and $t$ exchanges generate nonrenormalizable operators in the LESM in a calculable way. Thus $\bar{u} \gamma^{\mu}\left(1-\gamma^{5}\right) d \bar{e} \gamma_{\mu}\left(1-\gamma^{5}\right) \nu_{e}$, mediating neutron $\beta$ decay, is generated by $W$ exchange; $\bar{u} \gamma^{\mu}\left(1-\gamma^{5}\right) s \bar{d} \gamma_{\mu}\left(1-\gamma^{5}\right) u$, mediating $K^{-} \rightarrow \pi^{-} \pi^{0}$, is generated by $W$ exchange and hard gluon loops; and $\bar{b} \gamma^{\mu}\left(1-\gamma^{5}\right) d \bar{b} \gamma_{\mu}\left(1-\gamma^{5}\right) d$, mediating $B^{0}-\bar{B}^{0}$ mixing, is generated by $W-t$ box diagrams.

The interesting question, of course, is whether virtual $W, Z$ and $t$ exchange can generate all flavor-changing nonrenormalizable interactions in the LESM. And if the answer is no, which we hope and expect it to be, then with what precision must we do experiments in order to see deviations from this simple description?

There exist many models containing new particles and interactions with masses in the range $100 \mathrm{GeV}<M<1 \mathrm{TeV}$. A popular current framework for such physics beyond the Standard Model is supersymmetry, which in its minimal realization predicts 
a wealth of new degrees of freedom in this region. There are squarks and sleptons, gauginos, and charged and neutral Higgs bosons and their superpartners. The exchange of these heavy particles can have the effect of changing the coefficients of operators in the LESM which were already generated by $W, Z$ and $t$ exchange; for example, $\tilde{t}-\widetilde{w}$ box diagrams generate a contribution to the operator $\bar{b} \gamma^{\mu}\left(1-\gamma^{5}\right) d \bar{b} \gamma_{\mu}\left(1-\gamma^{5}\right) d$ responsible for $B^{0}-\bar{B}^{0}$ mixing. They can also have the effect of generating new nonrenormalizable operators in the LESM; for example, charged Higgs exchange generates the operator $\bar{c} b \bar{\tau} \nu_{\tau}$, which would provide a scalar component to semileptonic $B$ decay. Although it is certainly not the only possibility, the Minimal Supersymmetric Standard Model (MSSM) provides a convenient paradigm for exploring the variety of new interactions which new physics might induce at low energies [6].

\section{The quark sector of the Standard Model}

If we are to look in the LESM for a sign of new physics such as the MSSM, the Standard Model contributions must be well understood. From here on, we will concentrate on flavor changing processes in the quark sector, which has a particularly rich phenomenology. (With the recent strong experimental hints for neutrino masses and mixing, the phenomenology of the lepton sector is becoming interesting, too [7].) There are three quark $S U(2)$ doublets, which written in the mass eigenstate basis are

$$
\left(\begin{array}{l}
u \\
d
\end{array}\right), \quad\left(\begin{array}{l}
c \\
s
\end{array}\right), \quad\left(\begin{array}{l}
t \\
b
\end{array}\right) .
$$

The neutral current interactions of the quarks with the $\gamma$ and $Z$ (and with the gluon) are flavor-diagonal by the GIM mechanism. The flavor-changing interactions are mediated only by charged current interactions with the $W^{ \pm}$, of the form

$$
\left(\begin{array}{lll}
\bar{u} & \bar{c} & \bar{t}
\end{array}\right) \gamma^{\mu}\left(1-\gamma^{5}\right) V_{\mathrm{CKM}}\left(\begin{array}{c}
d \\
s \\
b
\end{array}\right) W_{\mu}+\text { h.c. } .
$$

The $3 \times 3$ unitary matrix $V_{\mathrm{CKM}}$, due to Cabibbo, Kobayashi and Maskawa [8, 9], is

$$
V_{\mathrm{CKM}}=\left(\begin{array}{ccc}
V_{u d} & V_{u s} & V_{u b} \\
V_{c d} & V_{c s} & V_{c b} \\
V_{t d} & V_{t s} & V_{t b}
\end{array}\right)
$$

The elements of $V_{\text {CKM }}$ have a hierarchical structure, getting smaller away from the diagonal: $V_{u d}, V_{c s}$ and $V_{t b}$ are of order $1, V_{u s}$ and $V_{c d}$ are of order $10^{-1}, V_{c b}$ and $V_{t s}$ are of order $10^{-2}$, and $V_{u b}$ and $V_{t d}$ are of order $10^{-3}$. The parameters of $V_{\mathrm{CKM}}$ originate

in the couplings of the chiral quark fields to the sector of the theory that breaks the 
electroweak and global flavor symmetries and generates the quark masses. In the mimimal Standard Model, these are Yukawa couplings to the Higgs field $\phi$,

$$
\lambda_{u}^{i j} \bar{Q}_{L}^{i} \tilde{\phi} u_{R}^{j}+\lambda_{d}^{i j} \bar{Q}_{L}^{i} \phi d_{R}^{j}+\text { h.c. . }
$$

The 36 complex parameters $\lambda_{u}^{i j}$ and $\lambda_{d}^{i j}$ may be reduced to 10 independent ones by applying the $U(3) \times U(3) \times U(3) / U(1)_{B}$ global symmetries of the quark kinetic terms. In the mass basis, these 10 quantities are the six quark masses $\left(m_{u}, m_{d}, m_{s}, m_{c}, m_{b}, m_{t}\right)$, and four parameters which characterize $V_{\mathrm{CKM}}$, including a complex phase.

A particularly convenient parameterization of $V_{\mathrm{CKM}}$, due to Wolfenstein [10], is

$$
V_{\mathrm{CKM}}=\left(\begin{array}{ccc}
1-\frac{1}{2} \lambda^{2} & \lambda & A \lambda^{3}(\rho-\mathrm{i} \eta) \\
-\lambda & 1-\frac{1}{2} \lambda^{2} & A \lambda^{2} \\
A \lambda^{3}(1-\rho-\mathrm{i} \eta) & -A \lambda^{2} & 1
\end{array}\right)+\mathcal{O}\left(\lambda^{4}\right)
$$

The Wolfenstein parameterization exploits the hierarchy in $V_{\mathrm{CKM}}$ by expanding explicitly in the small parameter $\lambda=\sin \theta_{C} \simeq 0.22$. The other parameters, $A, \rho$ and $\eta$, are expected to be of order one. To this order in $\lambda, V_{\mathrm{CKM}}$ is approximately unitary,

$$
V_{\mathrm{CKM}} V_{\mathrm{CKM}}^{\dagger}=1+\mathcal{O}\left(\lambda^{4}\right)
$$

If necessary, one may keep higher order terms in the expansion; when this is done, it is often useful to define $\bar{\rho}=\rho\left(1-\frac{1}{2} \lambda^{2}\right)$ and $\bar{\eta}=\eta\left(1-\frac{1}{2} \lambda^{2}\right)$.

There is an unremovable complex phase in $V_{\mathrm{CKM}}$ when $\eta \neq 0$. This phase induces complex couplings in the charged current interactions, which leads to the possibility that $\mathrm{CP}$ symmetry is violated. Because $\mathrm{CP}$ violation is a purely quantum phenomenon, it can be observed only in the interference between different quark-level amplitudes which mediate the same physical process. From the point of view of the LESM, there are two consequences. First, the coefficients of the nonrenormalizable flavor-changing operators must be taken to be complex numbers. Second, as a consequence of these unremovable complex phases, $\mathrm{CP}$ violation is something which is naturally present in fundamental interactions. It is not a mystery. The only question is whether it occurs at the level which one would expect in the Standard Model.

The phases of the elements of $V_{\mathrm{CKM}}$ in the Wolfenstein parameterization are

$$
\left(\begin{array}{ccc}
1 & 1 & \mathrm{e}^{-\mathrm{i} \gamma} \\
1 & 1 & 1 \\
\mathrm{e}^{-\mathrm{i} \beta} & 1 & 1
\end{array}\right)+\mathcal{O}\left(\lambda^{2}\right)
$$

which defines the angles $\beta$ and $\gamma$. Note that only the smallest elements $V_{u b}$ and $V_{t d}$ have phases of order one. The other matrix elements have small imaginary parts which appear only if $V_{\mathrm{CKM}}$ is expanded to higher order in $\lambda$. For example, $\arg V_{t s}=\lambda^{2} \eta$ is neglected at lowest order. As first pointed out by Kobayashi and Maskawa [9], and 
as is clear from this parameterization, $\mathrm{CP}$ violation involves the interference between amplitudes involving all three quark generations. Because of the smallness of $V_{u b}$ and $V_{t d}$, this phenomenon is inherently suppressed and only observable in particular experimental situations.

The violation of CP symmetry was first observed in neutral $K$ decays in 1964 by Cronin and Fitch. More precisely, what was observed was CP violation in $K^{0}-\bar{K}^{0}$ mixing, inducing a small CP-even component of the $K_{L}$ and thereby allowing the transition $K_{L} \rightarrow \pi \pi$. Mixing in the $K$ system is mediated by a $\Delta S=2$ operator, $\bar{s} \gamma^{\mu}\left(1-\gamma^{5}\right) d \bar{s} \gamma^{\mu}\left(1-\gamma^{5}\right) d$, which is generated in the Standard Model by box diagrams involving virtual $W^{\prime}$ 's and $u, c$, and $t$ quarks. The coefficient of the $\Delta S=2$ operator is of the form $A V_{c d}^{2} V_{c s}^{* 2}+B V_{t d}^{2} V_{t s}^{* 2}+C V_{c d} V_{t d} V_{c s}^{*} V_{t s}^{*}$, for constants $A, B, C$. Although the terms proportional to $B$ and $C$ are suppressed by powers of $\lambda$, they grow quickly with $m_{t}$ and their contribution is important. The violation of $\mathrm{CP}$ depends on the fact that the overall coefficient is complex, so the $t$-mediated terms which bring in $V_{t d}$ play the key role. The magnitude and phase of the phenomenological parameter $\epsilon_{K}$ which characterizes $\mathrm{CP}$ violation in mixing have been measured very precisely: $\left|\epsilon_{K}\right|=[2.258 \pm 0.018] \times 10^{-3}$ and $\arg \epsilon_{K}=(43.49 \pm 0.08)^{\circ}$ [11]. However, predicting $\epsilon_{K}$ in the Standard Model is problematic, because one needs not only the coefficient of the $\Delta S=2$ operator but also its matrix element, $\left\langle\bar{K}^{0}\left|\bar{s} \gamma^{\mu}\left(1-\gamma^{5}\right) d \bar{s} \gamma^{\mu}\left(1-\gamma^{5}\right) d\right| K^{0}\right\rangle$. Theoretical calculations of this matrix element rely on lattice QCD, and uncertainties are at the level of $20 \%$. What can be said now is only that $\epsilon_{K}$ is at the right level to come from the phase in $V_{\text {CKM }}$. However, this is still a significant observation!

More than three decades later, this year has seen the definitive observation of $\mathrm{CP}$ violation in the $\Delta S=1$ sector of the LESM as well. The operators in question are generated in the Standard Model by strong and electroweak penguin diagrams, the most important of which are

$$
\begin{aligned}
& Q_{6}=\bar{s}_{\alpha} \gamma^{\mu}\left(1-\gamma^{5}\right) d_{\beta} \sum_{q=u, d, s} \bar{q}_{\beta} \gamma_{\mu}\left(1-\gamma^{5}\right) q_{\alpha}, \\
& Q_{8}=\bar{s}_{\alpha} \gamma^{\mu}\left(1-\gamma^{5}\right) d_{\beta} \sum_{q=u, d, s} e_{q} \bar{q}_{\beta} \gamma_{\mu}\left(1-\gamma^{5}\right) q_{\alpha}
\end{aligned}
$$

where $\alpha, \beta$ are color indices. Since penguins can include intermediate $t$ quarks, the operator coefficients $C_{6}$ and $C_{8}$ receive complex contributions proportional to $\operatorname{Im} V_{t d} V_{t b}^{*}$. Both $Q_{6}$ and $Q_{8}$ mediate the transition $K \rightarrow \pi \pi$, and because $C_{6}$ and $C_{8}$ are complex, they mediate the CP violating transition $K_{L} \rightarrow \pi \pi$. The phenomenological parameter $\epsilon^{\prime}$ measures the extent to which CP violation differs in the $\Delta I=\frac{1}{2}$ and $\Delta I=\frac{3}{2}$ channels, and a nonzero $\epsilon^{\prime}$ is a signal that $\mathrm{CP}$ violation in the neutral $K$ system cannot be explained by mixing alone (in which case it would be independent of the final state).

This year, both KTeV $\left(\operatorname{Re}\left[\epsilon^{\prime} / \epsilon_{K}\right]=(28.0 \pm 4.1) \times 10^{-4}\right.$ [1] $)$ and NA48 $\left(\operatorname{Re}\left[\epsilon^{\prime} / \epsilon_{K}\right]=\right.$ $\left.(18.5 \pm 7.3) \times 10^{-4}[2]\right)$ confirmed the earlier NA31 result $\left(\operatorname{Re}\left[\epsilon^{\prime} / \epsilon_{K}\right]=(23 \pm 7) \times\right.$ 
$10^{-4}$ [3]) of a nonzero $\epsilon^{\prime} / \epsilon_{K}$. As with $\epsilon_{K}$, however, the prediction of $\epsilon^{\prime} / \epsilon_{K}$ in the Standard Model is problematic due to hadronic uncertainties. The matrix elements $\left\langle\pi \pi\left|Q_{6}\right| K_{L}\right\rangle$ and $\left\langle\pi \pi\left|Q_{6}\right| K_{L}\right\rangle$ are not related to each other, because of their different isopsin properties. In a very crude approximation which highlights the role of hadronic uncertainties, one may write 12

$$
\operatorname{Re}\left[\epsilon^{\prime} / \epsilon_{K}\right] \approx\left[B_{6}^{1 / 2}-0.5 B_{8}^{3 / 2}\right] \times 10^{-3}
$$

where the $B_{i}$ are "bag factors" which parametrize the matrix elements. A guess based on the vacuum insertion ansatz would yield $B_{6}^{1 / 2}=1.0, B_{8}^{3 / 2}=0.8$, and thus $\operatorname{Re}\left[\epsilon^{\prime} / \epsilon_{K}\right] \approx 7 \times 10^{-4}$, smaller by a factor of three than the experimental average. This has led some to speculate that the Standard Model cannot accommodate such a large effect. However, it cannot be stressed strongly enough that the hadronic uncertainties are large and hard to quantify, and that the values of $B_{i}$ taken from vacuum insertion easily could be off by a factor of two or more in either direction. It is worth noting in this context that the closely related hadronic matrix elements which account for the $\Delta I=\frac{1}{2}$ rule in $K$ decays remain very poorly understood. Under the present circumstances, there is not even a hint of new physics in this measurement.

In fact, what we have learned from the neutral $K$ system is not that the Standard Model is ruled out, but that it is confirmed: CP violation is at the level which one would expect. There is strong evidence for the correctness of the Standard Model picture that CP violation arises from the complex phase in $V_{\mathrm{CKM}}$, although hadronic uncertainties preclude precision tests as yet. In fact, the time has come to adopt the point of view that $\mathrm{CP}$ violation is a natural part of the Standard Model, and that as a phenomenon it is no longer particularly interesting for its own sake. Of course, there do remain crucial questions. Are there sources of $\mathrm{CP}$ violation beyond those from $V_{\mathrm{CKM}}$, and how can we observe them? Can CP violation be a tool for exploring the breaking of flavor symmetries in some more fundamental theory? To address these important issues, it is necessary to explore the nonrenormalizable operators of the LESM in as much variety and detail as possible.

\section{Constraints on $V_{\mathrm{CKM}}$}

We already have quite a lot of experimental information on the parameters of $V_{\mathrm{CKM}}$, interpreting the current data within the framework of the Standard Model. In this section I review the current constraints, which have changed little in the past year.

The best known element of $V_{\mathrm{CKM}}$ is $V_{u s}=\lambda=\sin \Theta_{C}$, the Cabibbo angle. It is proportional to the coefficient of the LESM operator $\bar{u} \gamma^{\mu}\left(1-\gamma^{5}\right) s \bar{e} \gamma_{\mu}\left(1-\gamma^{5}\right) \nu_{e}$, which mediates the well measured semileptonic decay $K^{-} \rightarrow \pi^{0} e^{-} \bar{\nu}_{e}$. To relate the rate for this process to $\lambda$, one needs to know the hadronic matrix element $\left\langle\pi^{0}\left|\bar{u} \gamma^{\mu}\left(1-\gamma^{5}\right) s\right| K^{-}\right\rangle$. 
Fortunately, in the chiral $S U(3)$ limit this is the matrix element of a globally conserved current, which can be computed exactly. Furthermore, chiral perturbation theory can be used to control higher order corrections in $m_{s}$, so the hadronic physics is under very good control [13]. From the measured rate for $K^{-} \rightarrow \pi^{0} e^{-} \bar{\nu}_{e}$, one finds [14]

$$
\lambda=0.2196 \pm 0.0023,
$$

an accuracy of about one percent.

The second best known element of $V_{\text {CKM }}$ is $V_{c b}=A \lambda^{2}$. This constant is the coefficient of the LESM operator $\bar{c} \gamma^{\mu}\left(1-\gamma^{5}\right) b \bar{e} \gamma_{\mu}\left(1-\gamma^{5}\right) \nu_{e}$, responsible for semileptonic $B$ meson decays. Both the exclusive decay $\bar{B} \rightarrow D^{*} \ell \bar{\nu}$ and the inclusive decay $\bar{B} \rightarrow X_{c} \ell \bar{\nu}$ have been used to extract $V_{c b}$, yielding consistent results. In both cases, the Heavy Quark Effective Theory, based on the approximation $m_{b} \rightarrow \infty$, is used to control the hadronic physics [15]. Combining the various experimental determinations, one finds

$$
V_{c b}=0.040 \pm 0.002 \quad \Rightarrow \quad A=0.83 \pm 0.04 .
$$

Theoretical uncertainties dominate the quoted error. The prospects for increasing the accuracy with which $A$ is known are summarized nicely in Ref. [15].

With $\lambda$ and $A$ reasonably well determined, $\rho$ and $\eta$ remain the most important unknowns in $V_{\mathrm{CKM}}$. It is convenient to plot the point $\rho+\mathrm{i} \eta$ in the complex plane. The triangle which is made by connecting the points $(0,0),(\rho, \eta)$ and $(1,0)$ is known as the "Unitarity Triangle", because its closure may be related to the unitarity of $V_{\text {CKM }}$. The angles of the Unitarity Triangle are labeled as shown in Fig. 目; on the west side of the Pacific Ocean, they are known as $\left(\phi_{1}, \phi_{2}, \phi_{3}\right)$, and on the east side they are known respectively as $(\beta, \alpha, \gamma)$. The unitarity of $V_{\mathrm{CKM}}$ is often expressed as the condition $\alpha+\beta+\gamma=\pi$. When $V_{\text {CKM }}$ is expanded to higher order in $\lambda$, what is plotted is often the point $\bar{\rho}+\mathrm{i} \bar{\eta}$ rather than $\rho+\mathrm{i} \eta$.

There are three important constraints on $\rho$ and $\eta$. The first is from the measurement of $\epsilon_{K}$. The computation of the box diagram which generates the LESM operator $\bar{s} \gamma^{\mu}\left(1-\gamma^{5}\right) d \bar{s} \gamma^{\mu}\left(1-\gamma^{5}\right) d$ responsible for $K^{0}-\bar{K}^{0}$ mixing can include intermediate charm and top quarks, and so the coefficient is a complicated combination of $V_{\mathrm{CKM}}$ parameters. To lowest order in $\lambda,\left|\epsilon_{K}\right|$ may be written as [16]

$$
\left|\epsilon_{K}\right|=3.8 \times 10^{4} \cdot B_{K} \cdot A^{2} \lambda^{6} \cdot \eta\left[f\left(x_{c}, x_{t}\right)+A^{2} \lambda^{4}(1-\rho) g\left(x_{t}\right)\right],
$$

where the short-distance quantities $f\left(x_{c}, x_{t}\right) \approx 7.2 \times 10^{-4}$ and $g\left(x_{t}\right) \approx 1.35$ are functions of $x_{c, t}=m_{c, t}^{2} / M_{W}^{2}$. They have been computed to next-to-leading order in QCD. The two terms in Eq. (14) are of roughly the same magnitude because of the strong dependence of the box diagram on $x_{t}$. The result is a hyperbola in the $(\rho, \eta)$ plane. However, the bag factor $B_{K}$, which parameterizes the hadronic matrix element 


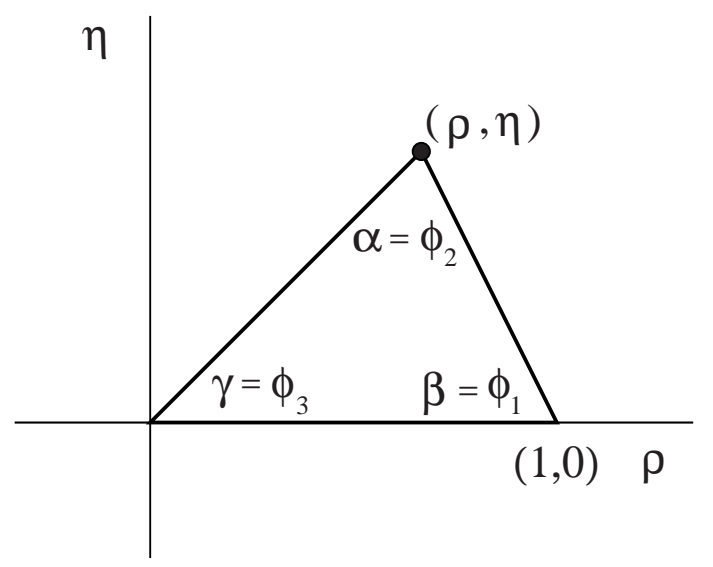

Figure 1: The unitarity triangle.

$\left\langle\bar{K}^{0}\left|\bar{s} \gamma^{\mu}\left(1-\gamma^{5}\right) d \bar{s} \gamma_{\mu}\left(1-\gamma^{5}\right) d\right| K^{0}\right\rangle=\frac{8}{3} m_{K}^{2} f_{K}^{2} B_{K}$, is not known precisely. Lattice QCD estimates yield approximately the range $0.6<B_{K}<1.0$, but the dominant uncertainties are from quenching and have not been quantified reliably [17]. Also, note that the fourth power of $A$ appears in the second term of Eq. (14), so the $5 \%$ experimental uncertainty on $A$ is magnified considerably.

The second constraint comes from the measurement of $\left|V_{u b} / V_{c b}\right|=\lambda \sqrt{\rho^{2}+\eta^{2}}$. The parameter $V_{u b}$ is most easily extracted from semileptonic $B$ decays, since it is proportional to the coefficient of the LESM operator $\bar{u} \gamma^{\mu}\left(1-\gamma^{5}\right) b \bar{\ell} \gamma_{\mu}\left(1-\gamma^{5}\right) \nu_{\ell}$. An important experimental difficulty is that there is a huge background from the process $b \rightarrow c \ell \bar{\nu}_{\ell}$, which in the Standard Model is approximately 100 times larger. Rejecting this background requires one to restrict the analysis either to a specific hadronic final state or to a small fraction of the lepton phase space. As will be discussed in Section 6.2.4, either approach introduces significant model dependence into the hadronic physics, with uncertainties which are very hard to quantify meaningfully. Taking the current central values but with a reasonably conservative attitude toward the theoretical errors, one has $\left|V_{u b} / V_{c b}\right|=0.090 \pm 0.025$ [15]. Analyses with subtantially smaller theoretical uncertainties should be taken with a grain of salt.

The third important constraint comes from $B^{0}-\bar{B}^{0}$ mixing, which is mediated by the LESM operator $\bar{b} \gamma^{\mu}\left(1-\gamma^{5}\right) d \bar{b} \gamma^{\mu}\left(1-\gamma^{5}\right) d$. In the Standard Model, this operator is generated by $t-W$ box diagrams, with a coefficient proportional to $\left|V_{t d}{ }^{*} V_{t b}\right|^{2}$. The phenomenological parameter $\Delta m_{d}$ is precisely measured, $\Delta m_{d}=$ $0.464 \pm 0.018 \mathrm{ps}^{-1}$ [14]. However, as in the case of $K^{0}-\bar{K}^{0}$ mixing, relating this number to fundamental quantities requires hadronic matrix elements which are difficult to compute. At leading order in $\lambda$ and next-to-leading order in QCD, one finds [16]

$$
\Delta m_{d}=1.30 G_{F}^{2} M_{W}^{2} / 6 \pi^{2} \cdot m_{B_{d}} \cdot f_{B_{d}}^{2} B_{B_{d}} \cdot A^{2} \lambda^{6} \cdot\left[(1-\rho)^{2}+\eta^{2}\right] .
$$


The quantity $f_{B_{d}}^{2} B_{B_{d}}$ parameterizes the matrix element. It is most accurately computed on the lattice, where $f_{B_{d}} \sqrt{B_{B_{d}}}$ has an uncertainty at the level of $20 \%$ [17.

In this case, the hadronic uncertainties can be reduced if the analagous quantity $\Delta m_{s}$ can be measured in $B_{s}-\bar{B}_{s}$ mixing. Then one can form a ratio,

$$
\frac{\Delta m_{d}}{\Delta m_{s}}=\xi^{-2} \frac{\left|V_{t d}\right|^{2}}{\left|V_{t s}\right|^{2}}=\xi^{-2} \cdot \lambda^{2}\left[(1-\rho)^{2}+\eta^{2}\right],
$$

where $\xi=\left[f_{B_{s}}^{2} B_{B_{s}} / f_{B_{d}}^{2} B_{B_{d}}\right]^{1 / 2}$. The ratio $\xi$ is unity in the $S U(3)$ limit. It can be studied much more reliably on the lattice than can either the numerator or the denominator, although calculations are still done only in the quenched theory. Recent estimates which attempt to include quenching errors give $\xi=1.14 \pm 0.13$ [17]. The current limit on $B_{s}-\bar{B}_{s}$ mixing, $\Delta m_{s}>12.4 \mathrm{ps}^{-1}$ [1], already contributes to constraining $(\rho, \eta)$. As the lower bound on $\Delta m_{s}$ increases (preliminary reports at this conference indicate $\Delta m_{s}>14.3 \mathrm{ps}^{-1}$ at $95 \%$ C.L. [18]), this constraint will become ever more important.

Combining the limits in the $(\rho, \eta)$ plane from $\epsilon_{K},\left|V_{u b}\right|, \Delta m_{d}$ and $\Delta m_{s}$ requires a consistent treatment of experimental and theoretical errors. This is problematic because the dominant theoretical hadronic uncertainties are difficult to quantify meaningfully and are certainly not in any sense Gaussian distributed about their "central values". The BaBar collaboration has proposed that a scanning method be used to deal with the theoretical errors associated with $B_{K}, f_{B_{d}}^{2} B_{B_{d}}, \xi$, and the extraction of $V_{u b}$. In this approach, the global fit to the data is repeated for a range of values of the theoretical inputs, and then the various allowed regions are overlayed to obtain an overall constraint. The BaBar Physics Book uses the fairly conservative ranges $B_{K}=0.6-1.0, f_{B_{d}}^{2} B_{B_{d}}=160-240 \mathrm{MeV}, \xi=1.06-1.22$, and $\left|V_{u b} / V_{c b}\right|=0.06-0.10$ [19]. Their allowed region for $(\rho, \eta)$, at $95 \%$ C.L., is reproduced in Fig. 2.

Other, more restrictive, regions in the $(\rho, \eta)$ plane have appeared in the literature [20]. However, it is important to note that they differ from the one shown here almost entirely in a less conservative treatment of theoretical uncertainties. With the exception of new determinations of $V_{u b}$, which are consistent with previous measurements and with comparable errors, there has been no recent change in the experimental input into the constraints. Until the lattice calculations of hadronic matrix elements improve, with quenching corrections under serious control, a conservative treatment of theoretical uncertainties is appropriate. The prospects for reducing the model dependence in the extraction of $V_{u b}$ will be discussed below; here both theory and experiment could contribute to improvement in the situation. Until there are future developments, Fig. 2 remains a reasonable representation of the state of our knowledge of $V_{\text {CKM }}$. 


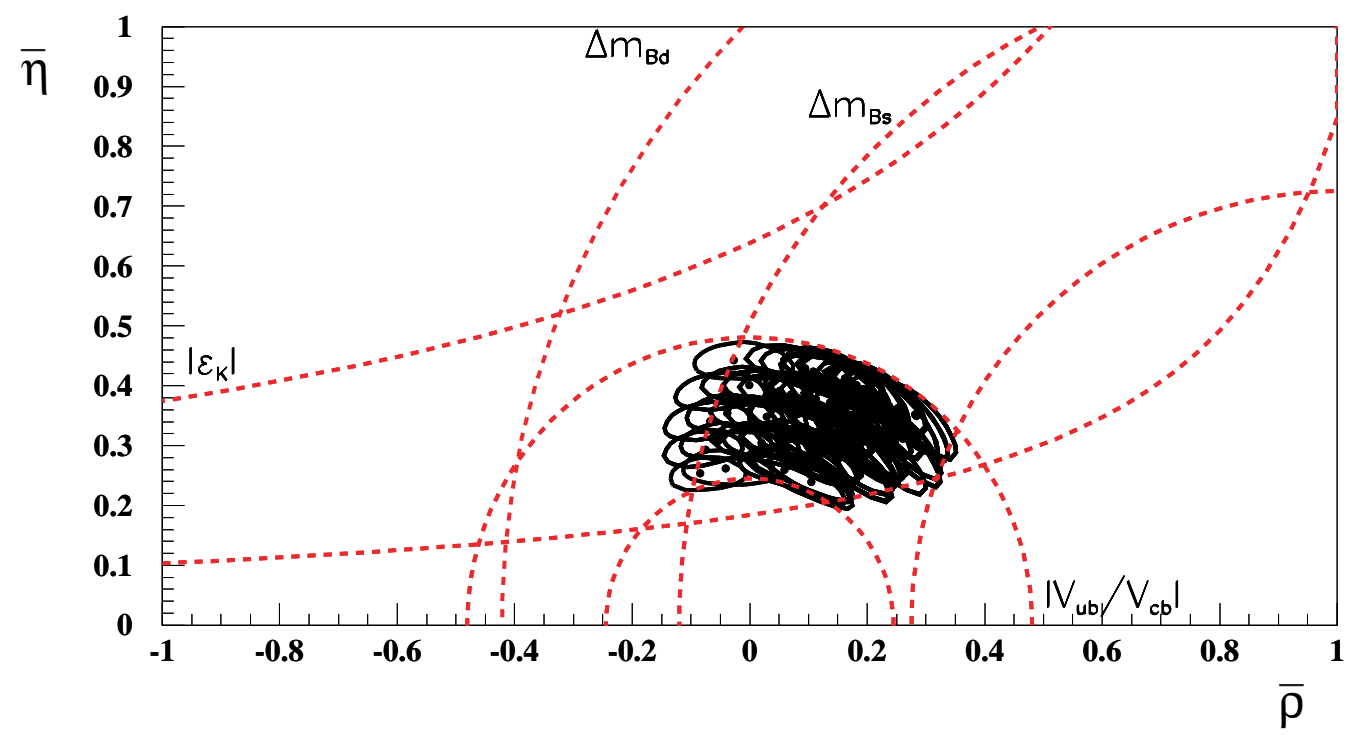

Figure 2: Constraints on the unitarity triangle, taken from The Babar Physics Book, 1998 [19]. The constraints are presented in the $(\bar{\rho}, \bar{\eta})$ plane, in which subleading corrections of relative order $\lambda^{2}$ have been included. Theoretical uncertainties are treated with a scanning method.

\section{$5 \quad$ Electroweak baryogenesis}

An interesting motivation to study CP violation in the Standard Model is the possible connection to the generation of the cosmological asymmetry between matter and antimatter. The net baryon-to-photon ratio in the universe is small but almost certainly nonzero, $\left(n_{B}-n_{\bar{B}}\right) \simeq 3 \times 10^{-10}$. While it is possible that this asymmetry is an initial condition of the Big Bang, it would be more satisfying if it could be explained as arising dynamically from matter symmetric initial conditions. It would be even more satisfying if the dynamics could be understood in terms of physical processes which already are known to occur in the early universe.

Sakharov identified three conditions which must be satisfied simultaneously for a baryon number asymmetry to be generated dynamically [21]. First, at some point the universe must depart from a state of thermal equilibrium. Second, at this time there must be unsuppressed processes which violate baryon number. Third, there must be $\mathrm{C}$ and $\mathrm{CP}$ violation in the theory, to allow baryons and antibaryons to be created at different rates. Finally, given these ingredients there also must exist a mechanism to generate the asymmetry at the right level.

It turns out that the Standard Model itself satisfies Sakharov's conditions during 
the electroweak phase transition [22]. At temperatures $k_{B} T \simeq 100 \mathrm{GeV}$, the Higgs sector settles into its ground state and assumes a vacuum expectation value $\langle\phi\rangle=$ $v=246 \mathrm{GeV}$, breaking the electroweak gauge symmetry $S U(2)_{L} \times U(1)_{Y} \rightarrow U(1)_{E M}$. If the phase transition is first order, it will proceed by the nucleation of bubbles of "true" vacuum, $\langle\phi\rangle=v$, in the "false" vacuum background with $\langle\phi\rangle=0$. The walls of the expanding bubbles will be sites of local thermal nonequilibrium, at which baryogenesis could take place. The baryon number violating interactions are provided by sphalerons, classical configurations of nonzero winding of the gauge and Higgs fields. Sphalerons are unsuppressed at high temperatures, when the electroweak symmetry is unbroken; at lower temperatures, they are suppressed by $\exp \left(-v / k_{B} T\right)$ and rapidly become unimportant as the universe cools. Finally, $\mathrm{C}$ is violated maximally in the Standard Model, while CP violation is supplied by $V_{\mathrm{CKM}}$.

Whether this mechanism is sufficient to produce the observed baryon number asymmetry is a detailed question which depends on the dynamics occurring at the bubble wall. In one scenario, the baryons are generated by a difference in scattering of left- and right-handed top quarks from the moving wall. The chiral asymmetry is then converted by sphalerons in the false vacuum into a net baryon number. Here it is crucial that there be enough $\mathrm{CP}$ violation in the quark-Higgs interactions. In addition, it is necessary that the asymmetry not be subsequently washed out by insufficiently suppressed sphaleron-mediated interactions in the bubble wall itself. This latter condition requires that the phase transition be strongly first order.

It turns out that the minimal Standard Model fails on both counts. First, because the effects of CP violation are suppressed both by the smallest couplings in $V_{\mathrm{CKM}}$ and by the quark masses, the "natural" size of the baryon asymmetry which could be generated is $10^{-21}$ [22]. To produce the observed asymmetry would require an enhancement of approximately eight orders of magnitude. Second, it is now known that the electroweak phase transition is not sufficiently first order to prevent sphalerons from eradicating the baryon number asymmetry once it is generated [22]. New physics is needed, if the asymmetry is to be produced at the electroweak phase transition.

Perhaps the most natural candidate for this new physics is supersymmetry. It is possible in the MSSM for the $\tilde{t}$ squark and the neutral and charged scalars $h$ and $H^{ \pm}$to be light, with masses in the range $100-200 \mathrm{GeV}$. In particular, if $100 \mathrm{GeV}<m_{\tilde{t}_{R}}<m_{t}$ and $m_{h}<115 \mathrm{GeV}$, then the phase transition can be sufficiently first order. There are also many new sources of CP violation in the MSSM. The most important of these for electroweak baryogenesis is the phase in the $H^{ \pm}$mass matrix. If this phase is of order one, and the $\tilde{t}$ and $H^{ \pm}$are light enough, then it remains possible to generate the observed baryon asymmetry 22].

However, even a large $\mathrm{CP}$ violating phase in the $H^{ \pm}$mass matrix will have small $\mathrm{CP}$ violating effects in $B$ decays. This is because the new phase is in the $\Delta B=0$ sector of the lagrangian. In such a scenario, it could be that the only effect in the $B$ system is a large contribution to $B^{0}-\bar{B}^{0}$ mixing from $\tilde{t}-\widetilde{w}$ box diagrams. This 
contribution could be as large as the $t-W$ box diagrams of the Standard Model; in this case, not only would the magnitude of $\Delta m_{d}$ be affected, but also its phase would change from its Standard Model value of $2 \beta$. Either of these effects, if large enough, could be identified experimentally.

In summary, then, electroweak baryogenesis should be considered as an indirect motivation for studying $\mathrm{CP}$ violation in the $B$ system. There are scenarios of electroweak baryogenesis which would leave a signature in $B^{0}-\bar{B}^{0}$ mixing, but there is no reason to expect that the new sources of $\mathrm{CP}$ violation which are needed to make these scnearios viable would manifest themselves directly in $B$ decays.

\section{Future scientific program}

The question to be addressed in the next round of experiments is whether the Standard Model can account completely for the flavor changing operators of the LESM. The hope, of course, is that the answer to this question is no, and that in seeing deviations from the Standard Model predictions we will get the first hints of new physics at the $\mathrm{TeV}$ scale.

What will be required to address this issue will be a robust program of $K$ and $B$ physics over the next decade. In particular, it would be ideal to measure $(\rho, \eta)$ independently in the $K$ and $B$ systems, through, respectively, the coefficients of $\Delta S=1,2$ and $\Delta B=1,2$ operators in the LESM. It will especially important that constraints on $\rho$ and $\eta$ be theoretically clean, in the sense that they be free of model-dependence and uncontrolled uncertainties associated with hadronic physics. Measurements which satisfy this criterion will have a crucial role to play in uncovering physics beyond the Standard Model.

\section{1 $K$ physics}

In the $K$ system, there are two experiments of particularly importance for constraining $V_{\mathrm{CKM}}$. The first is the measurement of the rate for the process $K^{-} \rightarrow \pi^{-} \nu \bar{\nu}$. This decay is mediated by the $\Delta S=1$ operator $\bar{d} \gamma^{\mu}\left(1-\gamma^{5}\right) s \bar{\nu} \gamma_{\mu}\left(1-\gamma^{5}\right) \nu$, which in the Standard Model is generated dominantly by penguin and box diagrams with virtual $t$ and $c$ quarks. The amplitude depends on a linear combination of $V_{t d}{ }^{*} V_{t s}$ and $V_{c d}{ }^{*} V_{c s}$. Because this is a semileptonic decay, the hadronic matrix element $\left\langle\pi^{-}\left|\bar{d} \gamma^{\mu}\left(1-\gamma^{5}\right) s\right| K^{-}\right\rangle$ can be related to the quantity $\left\langle\pi^{0}\left|\bar{u} \gamma^{\mu}\left(1-\gamma^{5}\right) s\right| K^{-}\right\rangle$measured in $K^{-} \rightarrow \pi^{0} e^{-} \bar{\nu}$ and is under good theoretical control. The largest uncertainty arises from computing the relative contribution from the virtual $c$ quark, and this is primarily because of the experimental uncertainty in $A$. An analysis at leading order in $\lambda$ and next-to-leading order in QCD yields the expression [16]

$$
\operatorname{BR}\left(K^{-} \rightarrow \pi^{-} \nu \bar{\nu}\right)=3.4 \times 10^{-4} \cdot A^{4} \lambda^{10} \cdot\left[\eta^{2}+\left(1+\delta_{c}-\rho\right)^{2}\right] .
$$


Hence a measurement of the rate would constrain an annulus centered at $(\rho, \eta)=$ $\left(1+\delta_{c}, 0\right)$ The charm contribution is

$$
\delta_{c}=1.5 \times 10^{-6} \cdot A^{-2} \lambda^{-4} \simeq 0.40 .
$$

It has a theoretical error of approximately $15 \%$, which is dominated by the remaining uncertainties in $A$ and, to a lesser extent, $\lambda$. Improvements in these quantities would also affect the normalization of the overall result.

The second key experiment would be to measure the rate for $K^{0} \rightarrow \pi^{0} \nu \bar{\nu}$. Except for a small component of higher angular momentum, this decay is purely CP violating, with an amplitude proportional to $\operatorname{Im} V_{t d}{ }^{*} V_{t s}=A^{2} \lambda^{5} \eta$. There is no contribution from intermediate charm quarks, and the calculation is theoretically very clean. At nextto-leading order in QCD, one has 16]

$$
\operatorname{BR}\left(K_{L} \rightarrow \pi^{0} \nu \bar{\nu}\right)=1.5 \times 10^{-3} \cdot A^{4} \lambda^{10} \cdot \eta^{2} .
$$

A measurement of the rate would constrain a horizontal band in the $(\rho, \eta)$ plane.

The only $\Delta S=2$ observable in the $K$ system is $\epsilon_{K}$; the difficulties in using it to constrain $V_{\text {CKM }}$ have been discussed in Section 1 . The theoretical expression for $\epsilon_{K}$ is given by Eq. (14). A reliable lattice computation of the bag parameter $B_{K}$ is desperately needed, but this will have to wait until the quenching corrections are understood quantitatively and reliably. A better determination of $A$ is also very important.

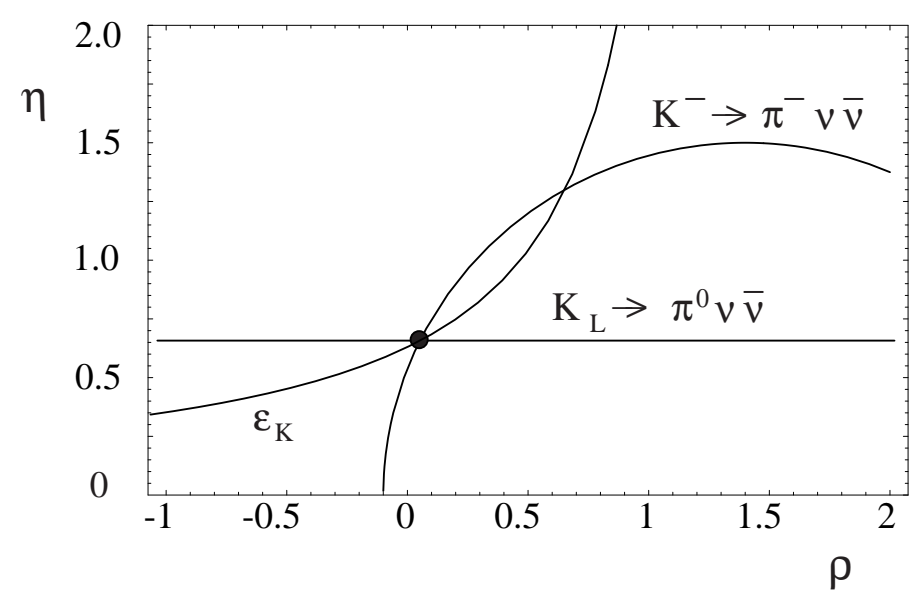

Figure 3: Hypothetical constraints on $(\rho, \eta)$ from measurements in the $K$ system.

The ability of $\operatorname{BR}\left(K^{-} \rightarrow \pi^{-} \nu \bar{\nu}\right), \operatorname{BR}\left(K_{L} \rightarrow \pi^{0} \nu \bar{\nu}\right)$ and $\epsilon_{K}$ to pinpoint $(\rho, \eta)$ using $K$ physics alone is illustrated in Fig. 3 . This is an idealized situation in which theoretical and experimental errors are neglected, but nevertheless it shows the power 
of the experiments on rare $K$ decays to constrain the Standard Model. As is apparent from the figure, of particular importance is the process $\operatorname{BR}\left(K_{L} \rightarrow \pi^{0} \nu \bar{\nu}\right)$. It should also be noted that the error in normalization from the remaining uncertainty in $A$ is largely correlated in the three quantities.

What are the experimental prospects for carrying out this program? In the mode $K^{ \pm} \rightarrow \pi^{ \pm} \nu \bar{\nu}$, the program is underway, as one event already has been observed at the Brookhaven expriment E787 [5]. The observation of this single event is consistent with the Standard Model expectation for the branching ratio at the level of $10^{-10}$. There is a proposal for a follow-on experiment at Brookhaven, E949, with the goal of seeing 10-20 events. A next generation experiment, CKM, has been proposed to run at a future Fermilab Main Injector fixed target program. If successful, CKM will make a $10 \%$ measurement of the branching ratio.

The mode $K_{L} \rightarrow \pi^{0} \nu \bar{\nu}$ is harder, both because the branching ratio is expected to be an order of magnitude smaller and because there are no charged particles in the final state. Proposals to attempt this measurement are being developed at Brookhaven (BNL-E926) and Fermilab (KAMI). The two proposals take different approaches to achieving the high level of background rejection which will be necessary for an experiment to be feasible. It is still unclear which approach, if either, has the better chance to succeed.

\section{2 $B$ physics}

The $B$ system offers many opportunities to constrain the Unitarity Triangle. There are two classes of measurements: those that measure the magnitudes of the elements of $V_{\mathrm{CKM}}$, such as $V_{u b}, V_{c b}, \Delta m_{d}$ and $\Delta m_{s}$, and those that measure directly the angles $\alpha, \beta$, and $\gamma$. The latter experiments probe $\mathrm{CP}$ violation in the $B$ system directly. Among their advantages is that in certain cases they can be free of uncertainties from hadronic physics. Rather than provide a broad survey of $B$ decay modes, which can be found in many places elsewhere (see, for example, Ref. [19]), I will focus on a few particularly important examples. In so doing, I will highlight what is meant by the measurement of a rate or an asymmetry being "theoretically clean".

The immediate future for $B$ physics experiments is extraordinarily exciting. This year saw the commissioning of two new asymmetric $B$ Factories operating at the $\Upsilon(4 S)$ resonance, PEP-II at SLAC and KEK-B at KEK. The luminosity goal for these accelerators, along with that of the upgraded symmetric collider CESR at Cornell, is in the range $10^{33}-10^{34} \mathrm{~cm}^{-2} \mathrm{sec}^{-1}$. The corresponding detectors, BaBar, BELLE and CLEO-III, will collect tens of millions of reconstructed $B$ decays. In addition, Run II at the Fermilab Main Injector will see luminosities of the order of $10^{33} \mathrm{~cm}^{-2} \mathrm{sec}^{-1}$ in $p \bar{p}$ collisions at $2 \mathrm{TeV}$, and with the substantial upgrades to $\mathrm{CDF}$ and D0, the hadron collider detectors will make important contributions as well. In particular, CDF expects to measure $\Delta m_{s}$ with high precision over and well beyond its entire 
Standard Model range.

In the longer term, there are proposals for dedicated $B$ experiments at the Tevatron $(\mathrm{BTeV})$ and the LHC (LHC-B). Such detectors, installed in the far forward region, would see enormous fluxes of all $b$ flavored hadrons. How best to do $B$ physics in such an active environment is currently the subject of intense study.

\subsubsection{CP asymmetry in $B \rightarrow J / \psi K_{S}$}

The "gold-plated" measurement in the $B$ system is the CP asymmetry in $B \rightarrow$ $J / \psi K_{S}$. Not only is the final state easy to identify and the branching ratio relatively large $\left(\sim 5 \times 10^{-4}\right)$, but the calculation of the asymmetry is theoretically quite clean. This measurement will be one of the early goals of all the $B$ Factory experiments.

The CP asymmetry arises from the interference between the direct decay $B^{0} \rightarrow$ $J / \psi K_{S}$ and the mixing-induced decay $B^{0} \rightarrow \bar{B}^{0} \rightarrow J / \psi K_{S}$. One can measure either a time-dependent or a time-integrated $\mathrm{CP}$ asymmetry. In the first case, one measures

$$
\begin{aligned}
a_{C P}(t) & =\frac{\Gamma\left(B_{\mathrm{phys}}^{0}(t) \rightarrow \psi K_{S}\right)-\Gamma\left(\bar{B}_{\mathrm{phys}}^{0}(t) \rightarrow \psi K_{S}\right)}{\Gamma\left(B_{\mathrm{phys}}^{0}(t) \rightarrow \psi K_{S}\right)+\Gamma\left(\bar{B}_{\mathrm{phys}}^{0}(t) \rightarrow \psi K_{S}\right)} \\
& =\frac{\left(1-\left|\lambda_{C P}\right|^{2}\right) \cos \left(\Delta m_{d} t\right)-2 \operatorname{Im} \lambda_{C P} \sin \left(\Delta m_{d} t\right)}{1+\left|\lambda_{C P}\right|^{2}} \\
& =-\operatorname{Im} \lambda_{C P} \sin \left(\Delta m_{d} t\right),
\end{aligned}
$$

where $\lambda_{C P}$ is a $\mathrm{CP}$ violating observable which depends on the final state, and the last line holds because in the case of $J / \psi K_{S}$ direct CP violation is negligible and therefore $\left|\lambda_{C P}\right|=1$. The notation $B_{\text {phys }}^{0}(t)$ refers to a state which is tagged as a $B^{0}$ at time $t=0$ and decays at time $t$. For the time-integrated asymmetry (which cannot be observed at the $\Upsilon(4 S)$ because of the quantum coherence of the initial $B^{0} \bar{B}^{0}$ state), one has, with $\left|\lambda_{C P}\right|=1$,

$$
a_{C P}=\frac{\Gamma\left(B^{0} \rightarrow \psi K_{S}\right)-\Gamma\left(\bar{B}^{0} \rightarrow \psi K_{S}\right)}{\Gamma\left(B^{0} \rightarrow \psi K_{S}\right)+\Gamma\left(\bar{B}^{0} \rightarrow \psi K_{S}\right)}=-\frac{x_{d}}{1+x_{d}^{2}} \operatorname{Im} \lambda_{C P},
$$

where $x_{d}=\Delta m_{d} / \Gamma\left(B_{d}\right)=0.72 \pm 0.03$.

The phase of $\lambda_{C P}$ is independent of the phase convention for $V_{\mathrm{CKM}}$ and has a straightforward relationship to the underlying process. Let $\mathcal{M}_{B}$ be the amplitude for $B^{0}-\bar{B}^{0}$ mixing, $\mathcal{A}_{\psi K_{S}}$ the amplitude for $B^{0} \rightarrow J / \psi K_{S}$, and $\mathcal{M}_{K}$ the amplitude for $K^{0}-\bar{K}^{0}$ mixing. Then

$$
\arg \lambda_{C P}=\arg \mathcal{M}_{B}+2 \arg \mathcal{A}_{\psi K_{S}}+\arg \mathcal{M}_{K}+\pi,
$$

where the extra $\pi$ arises because the final state $J / \psi K_{S}$ is CP-odd. In the Standard Model, $\mathcal{M}_{B}$ is dominated by $t-W$ box diagrams, and $\arg \mathcal{M}_{B}=2 \arg \left(V_{t d} V_{t b}^{*}\right)=-2 \beta$. 
Similarly, $\mathcal{M}_{K}$ is mediated by $c-W$ box diagrams, so $\arg \mathcal{M}_{K}=2 \arg \left(V_{c d} V_{c s}^{*}\right)=$ 0 . Finally, the dominant contribution to the decay amplitude is from the LESM operator $\bar{c} \gamma^{\mu}\left(1-\gamma^{5}\right) b \bar{s} \gamma_{\mu}\left(1-\gamma^{5}\right) c$, induced by tree level $W$ exchange, so $\arg \mathcal{A}_{\psi K_{S}}=$ $\arg \left(V_{c b} V_{c s}^{*}\right)=0$. Putting these elements together, we find $a_{C P} \propto \operatorname{Im} \lambda_{C P}=\sin 2 \beta$. Note that as long as the arguments of $\mathcal{M}_{K}$ and $\mathcal{A}_{\psi K_{S}}$ vanish as in the Standard Model, the asymmetry in $B \rightarrow J / \psi K_{S}$ measures the phase of $B^{0}-\bar{B}^{0}$ mixing.

The tree process is not the only one which can contribute to $\mathcal{A}_{\psi K_{S}}$. There are also strong penguin diagrams, which induce in the LESM the operator $\bar{s} \gamma^{\mu} T^{a} b \bar{c} \gamma_{\mu} T^{a} c$, where $T^{a}$ are $S U(3)$ matrices. The matrix elements $\left\langle\psi K_{S}\left|\bar{c} \gamma^{\mu}\left(1-\gamma^{5}\right) b \bar{s} \gamma_{\mu}\left(1-\gamma^{5}\right) c\right| B\right\rangle$ and $\left\langle\psi K_{S}\left|\bar{s} \gamma^{\mu} T^{a} b \bar{c} \gamma_{\mu} T^{a} c\right| B\right\rangle$ are independent hadronic quantities which are not related to each other by any symmetry. Therefore the ratio of the penguin-mediated amplitude to the tree amplitude is model-dependent and cannot as yet be estimated reliably. This is potentially a source of serious trouble, because it is absolutely indispensable to know accurately the weak phase of $\mathcal{A}_{\psi K_{S}}$, which depends on this ratio.

However, let us examine the penguin contribution more closely. The largest diagram has a virtual $t$ quark and is proportional to $V_{t b} V_{t s}^{*}$. Fortunately, though, $\arg \left(V_{t b} V_{t s}^{*}\right)=\mathcal{O}\left(\lambda^{2}\right)$ is almost the same as $\arg \left(V_{c b} V_{c s}^{*}\right)$. Since the penguin diagram is also loop suppressed by $\alpha_{s} / 4 \pi$, the phase of $\mathcal{A}_{\psi K_{S}}$ is largely unaffected by this term, regardless of the unknown ratio of hadronic matrix elements. The penguin diagram with a virtual $u$ quark has an effect of the same small size; its argument $-\gamma$ is of $\mathcal{O}(1)$, but its magnitude is suppressed by $\lambda^{2}$ compared to the $t$ penguin. In the end, penguin corrections to $\arg \mathcal{A}_{\psi K_{S}}$ are expected to be below the level of $10^{-2}$ and can be safely neglected. This is what is meant by the statement that $B \rightarrow J / \psi K_{S}$ is a "theoretically clean" mode from which to extract a CP asymmetry.

The current limits on $(\rho, \eta)$ place significant constraints on the values of $\sin 2 \beta$ which are consistent with the Standard Model. Treating theoretical uncertainties conservatively, one finds an allowed range of roughly $0.4<\sin 2 \beta<0.8$ [19]. Note that the sign of the asymmetry is predicted. The recent CDF result is

$$
\sin 2 \beta=0.79_{-0.44}^{+0.41}, \quad \text { with } \quad 0.00 \leq \sin 2 \beta<1.00 \quad \text { at } 93 \% \text { C.L. . }
$$

Although the errors are still large, the measurement is consistent with the indirect constraints, and most important, the sign comes out as expected. This result is actually a little disappointing, since if the sign had been negative then we would have had our first unambiguous indication of physics beyond the Standard Model.

Future measurements will constrain the asymmetry in $B \rightarrow J / \psi K_{S}$ much more tightly. The upcoming $B$ Factory experiments BaBar and BELLE, along with CDF and D0 at Run II and HERA-B, will succeed in achieving $\delta \sin 2 \beta=0.05-0.10$. Farther in the future, hadronic " $B$ Factories" should be able to identify this final state with little trouble and measure the asymmetry extremely well. BTeV and LHC-B, as well as ATLAS and CMS, expect an eventual accuracy of $\delta \sin 2 \beta=0.01-0.02$. 
With the theoretical prediction well under control and the experimental prospects so promising, this mode is "gold-plated", indeed!

\subsubsection{CP asymmetry in $B \rightarrow \pi^{+} \pi^{-}$}

Unfortunately, the same cannot be said of $B \rightarrow \pi^{+} \pi^{-}$, a mode once thought to be as useful as $B \rightarrow J / \psi K_{S}$ for constraining the Unitarity Triangle. An asymmetry analogous to (20) or (21) may be measured for the $\pi^{+} \pi^{-}$final state. The only difference in the analysis is that now

$$
\arg \lambda_{C P}=\arg \mathcal{M}_{B}+2 \arg \mathcal{A}_{\pi \pi}
$$

The tree level contribution to $\mathcal{A}_{\pi \pi}$ is proportional to $V_{u b} V_{u d}^{*}$. Since $\arg \left(V_{u b} V_{u d}^{*}\right)=-\gamma$, the asymmetry in $B \rightarrow \pi^{+} \pi^{-}$is proportional to $\operatorname{Im} \lambda_{C P}=-\sin (2 \beta+2 \gamma)=\sin 2 \alpha$, using the unitarity relation $\alpha+\beta+\gamma=\pi$.

The problem comes when we consider the penguin contributions to $\arg \mathcal{A}_{\pi \pi}$. The penguin diagram with an virtual $t$ quark in the loop yields a term in the amplitude with argument $\arg \left(V_{t b} V_{t d}^{*}\right)=\beta$ rather than $\arg \left(V_{u b} V_{u d}^{*}\right)=-\gamma$, but with no suppression by powers of $\lambda$. The tree operator $\bar{u} \gamma^{\mu}\left(1-\gamma^{5}\right) b \bar{d} \gamma_{\mu}\left(1-\gamma^{5}\right) u$ and the penguin operator $\bar{d} \gamma^{\mu} T^{a} b \bar{u} \gamma_{\mu} T^{a} u$ have different weak phases and hadronic matrix elements. The asymmetry in $B \rightarrow \pi^{+} \pi^{-}$still depends cleanly on the weak phase of $\mathcal{A}_{\pi \pi}$, but $\mathcal{A}_{\pi \pi}$ is now proportional to an unknown linear combination of $\mathrm{e}^{-\mathrm{i} \gamma}$ and $\mathrm{e}^{\mathrm{i} \beta}$. Absent reliable knowledge of both the relative magnitudes and strong phases of $\left\langle\pi^{+} \pi^{-}\left|\bar{u} \gamma^{\mu}\left(1-\gamma^{5}\right) b \bar{d} \gamma_{\mu}\left(1-\gamma^{5}\right) u\right| B\right\rangle$ and $\left\langle\pi^{+} \pi^{-}\left|\bar{d} \gamma^{\mu} T^{a} b \bar{u} \gamma_{\mu} T^{a} u\right| B\right\rangle$, even a precise measurement of the asymmetry cannot be used to extract cleanly the phase of any single operator in the LESM, nor the phase of any element of $V_{\mathrm{CKM}}$.

Only the loop factor $\alpha_{s} / 4 \pi$ remains to suppress the contribution of the penguin diagram. Since the decay $B \rightarrow K \pi$ is probably dominated by penguins (in this mode the tree contribution to $b \rightarrow u \bar{u} s$ is suppressed by $\lambda^{2}$ compared to the penguin contribution), it provides a probe of the strength of the penguin matrix elements. Recent data on $B \rightarrow K \pi$ which indicate that $\mathrm{BR}(B \rightarrow K \pi)>\mathrm{BR}(B \rightarrow \pi \pi)$ are not encouraging. Denoting by $|P / T|$ the relative contribution of penguin to tree processes in $B \rightarrow \pi \pi$, the data suggest $0.2<|P / T|<0.6$ [18, 19]. This is well in the range where the "penguin pollution" is fatal.

In principle, the penguin contributions can be disentangled by exploiting the isospin structure of the decay. The tree operator has both $\Delta I=\frac{1}{2}$ and $\Delta I=\frac{3}{2}$ components, while the penguin is pure $\Delta I=\frac{1}{2}$. Gronau and London observed that this could be done by measuring the flavor-tagged rates for $B \rightarrow \pi^{+} \pi^{-}, \pi^{0} \pi^{0}, \pi^{ \pm} \pi^{0}$ [23]. The experimental difficulty is with $B \rightarrow \pi^{0} \pi^{0}$ : not only is the branching ratio for this "color-suppressed" mode expected to be as small as $10^{-7}$, but the four-photon final state is extremely difficult to identify. So far, none of the current or proposed $B$ Factories has claimed to be able to make this measurement. 
An alternative explored by Quinn and Snyder is to do a Dalitz plot analysis of the $\mathrm{CP}$ asymmetry in $B \rightarrow\left(\rho^{-} \pi^{+}, \rho^{+} \pi^{-}, \rho^{0} \pi^{0}\right) \rightarrow \pi^{+} \pi^{-} \pi^{0}$ 24]. The various $\rho \pi$ intermediate states have different isospin quantum numbers and hence different sensitivity to the tree and penguin operators. An additional advantage of this approach is that one simultaneously would determine $\sin 2 \alpha$ and $\cos 2 \alpha$, reducing the ambiguity in extracting $\alpha$ itself. However, thousands of reconstructed events would be needed to achieve the necessary sensitivity. Whether either the $B$ Factories at the $\Upsilon(4 S)$ or the proposed hadronic $B$ experiments BTeV and LHC-B can do such an analysis remains an open and extremely important question.

\subsubsection{Direct $\mathrm{CP}$ violation in $B$ decays and the extraction of $\gamma$}

There is a wide variety of proposals to measure the third angle $\gamma$ of the Unitarity Triangle. In constrast to the other two angles, to measure $\gamma$ one must rely on direct $\mathrm{CP}$ violation in $B$ decays. This introduces strong phases in an essential way, with two important implications. First, often they must be extracted or bounded simultaneously with $\gamma$. Second, the ultimate sensitivity of a given construction to $\gamma$ typically depends on strong phases which are not known beforehand. Here I will mention very briefly two classes of proposals for measuring $\gamma$.

First, it is possible to extract $\gamma$ cleanly from rate measurements in $B^{ \pm} \rightarrow D K^{ \pm}$ and $B_{s} \rightarrow D_{s} K^{ \pm}$, but both experiments are difficult. The strategy in $B^{ \pm}$decay is to find two final states $f_{i}$, each common to $D^{0}$ and $\bar{D}^{0}$, and extract the strong and weak phases from the four decays $B^{ \pm} \rightarrow\left(D^{0}, \bar{D}^{0}\right) K^{ \pm} \rightarrow f_{i} K^{ \pm}$. The total branching ratios are at the level of $10^{-7}$ and high precision is required, so it is not clear whether the $B$ Factories at the $\Upsilon(4 S)$ will have sufficient statistics to complete such an analysis [19]. The second option, involving $B_{s}$ decays, certainly must wait for BTeV or LHC-B.

The second class of methods is to extract $\gamma$ from combinations of rates of $B \rightarrow K \pi$ modes. While there are many such approaches, all require additional inputs of some kind 25]. The most common of these are $S U(3)$ flavor symmetry and dynamical assumptions concerning rescattering effects, the sizes of penguin diagrams, or factorization of hadronic matrix elements. Unfortunately, there are too many such proposals to review them usefully here. Although none is as theoretically clean as are the extractions from $B_{(s)} \rightarrow D_{(s)} K^{ \pm}$rates, analyses of this sort may be interesting if one treats very conservatively the uncertainty from the additional assumptions [26].

\subsubsection{Measurement of $\left|V_{u b}\right|$}

Our current knowledge of $\left|V_{u b} / V_{c b}\right|=\lambda \sqrt{\rho^{2}+\eta^{2}}$ comes from semileptonic decays mediated by the tree level process $b \rightarrow u \ell \bar{\nu}$. Both inclusive $\bar{B} \rightarrow X_{u} \ell \bar{\nu}$ and exclusive $\bar{B} \rightarrow(\pi, \rho) \ell \bar{\nu}$ decays have been used to extract this parameter. All determinations of $\left|V_{u b}\right|$ must contend with an enormous background from $b \rightarrow c \ell \bar{\nu}$, which in the Standard Model is approximately 100 times larger. Inclusive analyses reject charmed final 
states by imposing strict kinematic cuts, such as $E_{\ell}>2.3 \mathrm{GeV}$ or $M\left(X_{u}\right)<1.8 \mathrm{GeV}$. (The mass $M\left(X_{u}\right)$ is inferred by reconstructing the missing neutrino.) However, these cuts have an unfortunate impact on the theoretical prediction of the decay rate.

The total rate is given at tree level by $\Gamma\left(\bar{B} \rightarrow X_{u} \ell \bar{\nu}\right)=G_{F}^{2} m_{b}^{5} / 192 \pi^{3} \cdot\left|V_{u b}\right|^{2}$, with radiative and nonperturbative corrections which are well understood [15]. The largest uncertainty comes from the value of $m_{b}$, which currently may be determined with an error of approximately $100 \mathrm{MeV}$. The induced theoretical uncertainty in $\left|V_{u b}\right|$ is at the level of $5 \%$. As stringent kinematic cuts are applied, however, the inclusive rate becomes much less inclusive, developing an essential dependence on the momentum distribution of the $b$ quark inside the $B$ meson. This is because in the presence of the cuts one becomes sensitive to the shape of the differential decay spectrum, not just to its integral. On scales of relative order $\Lambda_{\mathrm{QCD}} / m_{b}$, the spectrum probes all moments of this $b$ quark "wavefunction". Since the wavefunction is not known from first principles and must be modeled, this introduces an irreducible and uncontrolled model dependence into the analysis. The earliest determinations of $\left|V_{u b}\right|$ from the endpoint region in $E_{\ell}$ were completely polluted in this way, with results varying by as much as a factor of two from model to model.

More recent determinations rely primarily on the cut $M\left(X_{u}\right)<1.6 \mathrm{GeV}$, which keeps a significantly larger fraction of the charmless final state phase space than does a stringent cut on $E_{\ell}$ alone. A recent LEP average yields [27

$$
\left|V_{u b}\right|=\left[4.05_{-0.46}^{+0.39}(\text { stat. })_{-0.51}^{+0.43}(b \rightarrow c)_{-0.27}^{+0.23}(b \rightarrow u) \pm 0.02\left(\tau_{b}\right) \pm 0.16(\mathrm{HQE})\right] \times 10^{-3},
$$

or approximately $\left|V_{u b} / V_{c b}\right|=0.104_{-0.018}^{+0.015}$. While these analyses are experimentally very sophisticated, they rely intensively on a two-parameter model of the $b$ quark wavefunction. Essentially, in such a parameterization all moments of the $b$ momentum distribution are correlated with the first two nonzero ones, a constraint which is unphysical. Even if the two parameters are varied within "reasonable" ranges, it is doubtful that such a restrictive choice of model captures reliably the true uncertainty in $\left|V_{u b}\right|$ from our ignorance of the structure of the $B$ meson. While the central value which is obtained in these analyses is reasonable, the realistic theoretical error which should be assigned is not yet well understood.

A recent analysis by CLEO of the exclusive decay $\bar{B} \rightarrow \rho \ell \bar{\nu}$ yields [28]

$$
\left|V_{u b}\right|=\left[3.25 \pm 0.14 \text { (stat.) }{ }_{-0.29}^{+0.21} \text { (syst.) } \pm 0.55 \text { (theory) }\right) \times 10^{-3} \text {, }
$$

or approximately $\left|V_{u b} / V_{c b}\right|=0.083_{-0.016}^{+0.015}$, essentially consistent with the LEP result. In this case the reliance on models is quite explicit, since one needs the hadronic form factor $\left\langle\rho\left|\bar{u} \gamma^{\mu}\left(1-\gamma^{5}\right) b\right| \bar{B}\right\rangle$ over the range of momentum transfer to the leptons. The CLEO measurement relies on models based on QCD sum rules, which have uncertainties which are hard to quantify. Hence, just as in the case of the LEP measurement, the quoted errors should not be taken terribly seriously. All of the 
current constraints are consistent with $\left|V_{u b} / V_{c b}\right|=0.090 \pm 0.025$, where I strongly prefer this more conservative estimate of the theoretical errors. The problem lies not in the experimental analyses, but in our insufficient undertanding of hadron dynamics.

As is clear from Fig. 2, a reliable measurement of $\left|V_{u b}\right|$ would provide an important constraint in the $(\rho, \eta)$ plane. What needs to be done to improve the present situation? For analyses based on inclusive decays, the model dependence will be reduced only if the kinematic cuts used to reduce the charm contamination can be loosened. The larger the fraction of the phase space for $\bar{B} \rightarrow X_{u} \ell \bar{\nu}$ which is actually observed, the smaller will be the sensitivity to the shape of the decay spectrum. For exclusive analyses, a more reliable understanding of the form factors is needed. Eventually, the lattice should provide a good calculation of $\left\langle\rho\left|\bar{u} \gamma^{\mu}\left(1-\gamma^{5}\right) b\right| \bar{B}\right\rangle$; in this case, we are fortunate that the lattice computations are most reliable in the region of large momentum transfer to the leptons, where the experiments are also the most sensitive. Alternatively, one may use heavy quark and $S U(3)$ flavor symmetry to relate $\bar{B} \rightarrow \rho \ell \bar{\nu}$ to $D \rightarrow \bar{K}^{*} \ell \nu$. It will be crucial to understand the leading corrections from symmetry breaking effects [29]. Although the extraction of $\left|V_{u b}\right|$ from $B \rightarrow \pi \ell \bar{\nu}$ is more complicated due to the $B^{*}$ pole, dispersion relations possibly can be used to control the hadronic form factors [30]. Finally, there have been proposals to extract $\left|V_{u b}\right|$ from inclusive nonleptonic decays [31]. This method is under excellent theoretical control, but it remains to be seen whether it is feasible experimentally.

\subsection{5 $B$ physics tests of the Standard Model}

The ultimate goal of a robust $K$ and $B$ physics program is to probe the adequacy of the Standard Model description of the LESM by comparing many independent constraints in the $(\rho, \eta)$ plane. Yet there also exist simple strategies for looking for new physics in the $B$ system alone, which may prove fruitful long before the complete program can be realized. Here I will discuss briefly two simple examples.

The first is to compare the $\mathrm{CP}$ asymmetries in $B \rightarrow J / \psi K_{S}$ and $B \rightarrow \phi K_{S}$ [32]. In the Standard Model, both of these measure the phase of $B^{0}-\bar{B}^{0}$ mixing, since the decay amplitudes are real to a good approximation. For $B \rightarrow J / \psi K_{S}$, the decay is dominated by a tree level process $b \rightarrow c \bar{c} s$, which is large and therefore not so likely to receive significant contributions from new physics. By constrast, the $b \rightarrow s \bar{s} s$ transition underlying $B \rightarrow \phi K_{S}$ is generated only by penguin diagrams, so it carries a natural suppression of $\alpha_{s} / 4 \pi$. Because of this suppression, it is possible that loops with new heavy particles could contribute significantly to $b \rightarrow s \bar{s} s$ but not to $b \rightarrow c \bar{c} s$. If these new loops carried nonzero phases, then the value of "sin $2 \beta$ " extracted from $B \rightarrow \phi K_{S}$ would differ from that extracted from $B \rightarrow J / \psi K_{S}$. Such an observation would be an unambiguous and exciting sign of new physics.

The second example is a strategy for identifying new contributions to the phase of $B^{0}-\bar{B}^{0}$ mixing [33]. Note that if one compares Eqs. (22) and (24) for the asymmetries 
in $B \rightarrow J / \psi K_{S}$ and in $B \rightarrow \pi \pi$, the difference is independent of the mixing amplitude $\mathcal{M}_{B}$ and sensitive to

$$
2 \arg \mathcal{A}_{\psi K_{S}}+\arg \mathcal{M}_{K}-2 \arg \mathcal{A}_{\pi \pi}
$$

which is $2 \gamma$ in the Standard Model. If an isospin analysis can be done in $B \rightarrow$ $\pi \pi$ or $B \rightarrow \rho \pi$, the difference of asymmetries (25) can be combined with $\sqrt{\rho^{2}+\eta^{2}}$ from a measurement of $\left|V_{u b} / V_{c b}\right|$ to fix the point $(\rho, \eta)$. Once $(\rho, \eta)$ is identified, the angle $\beta$ can be predicted, and thus one knows the phase $\arg \mathcal{M}_{B}$ which should be extracted from $B \rightarrow J / \psi K_{S}$ if the mixing is given by Standard Model physics. New contributions to $B^{0}-\bar{B}^{0}$ mixing should show up in the comparison, since there is no reason for them to have the same nonzero phase as that predicted by $V_{\mathrm{CKM}}$. Such an analysis would be sensitive, for example, to mixing induced by supersymmetric $\tilde{t}-\widetilde{w}$ box diagrams, as expected in scenarios of electroweak baryogenesis in the MSSM.

\section{A few concluding comments}

A rich program of experimental and theoretical flavor physics awaits us in the first decade of the next century. One of the most delightful aspects of this field is the strong and growing coupling between theory and experiment. The complications brought by hadronic physics force the two sides of our community to work together to identify and develop analyses which are both feasible experimentally and controllable theoretically.

The most important goal in $K$ physics is to measure with $10 \%$ accuracy the branching ratios for the very rare decays $K^{-} \rightarrow \pi^{-} \nu \bar{\nu}$ and $K_{L} \rightarrow \pi^{0} \nu \bar{\nu}$. To do so will require both extensive $R \& D$ by physicists and continuing support from the funding agencies. But these experiments are crucial, and we must commit to them.

In $B$ physics, a host of new experiments awaits us. Over the next decade we will have a wide variety of measurements of $\mathrm{CP}$ violating asymmetries, branching fractions, and mixing parameters in the $B$ and $B_{s}$ systems. There are important roles to be played by all of the current and proposed detectors. In the next few years, the $B$ Factories at the $\Upsilon(4 S)$ will take the lead, along with the Tevatron experiments and HERA-B. But in the longer term it is crucial that there be a dedicated $B$ experiment at a high energy hadron collider, such as BTeV or LHC-B. Such a detector will be able to explore the $B_{s}$ system and observe some of the rarest $B$ decays. A luminosity upgrade to one of the $e^{+} e^{-} B$ Factories also may be in our future.

Finally, a few philosophical points. First, every measurement of a new operator in the Low Energy Standard Model is significant, especially if the measurement is redundant from the point of view of constraining the Unitarity Triangle. Such comparisons are how we will find new physics if it is there! Second, one theoretically clean measurement is worth many polluted ones, although even polluted ones will become important as our understanding of hadronic physics improves. Third, the goal of this 
program is not simply to measure $\rho$ and $\eta$, nor to constrain $\alpha, \beta$ and $\gamma$, nor to search for CP violation in the $B$ system. Rather, it is to learn as much as possible about the physics of flavor, and to probe robustly the Standard Model description of flavor changing dynamics. When new degrees of freedom are seen at Fermilab Run II or at the LHC, their virtual impact on "low energy" physics will be crucial in helping us to understand in detail what we have actually discovered.

This work was supported by the National Science Foundation under grant PHY9404057 and National Young Investigator Award PHY-9457916, the Department of Energy under Outstanding Junior Investigator Award DE-FG02-94ER40869, and the Alfred P. Sloan Foundation. A.F. is a Cottrell Scholar of the Research Corporation.

\section{References}

[1] A. Alavi-Harati et al. [KTeV Collaboration], Phys. Rev. Lett. 83, 22 (1999).

[2] P. Debu [NA48 Collaboration], CERN seminar, presented June 18, 1999, http://www.cern.ch/NA48.

[3] G.D. Barr et al. [NA31 Collaboration], Phys. Lett. B317, 233 (1993).

[4] F. Abe et al. [CDF Collaboration], Phys. Rev. Lett. 81, 5513 (1998).

[5] S. Adler et al. [E787 Collaboration], Phys. Rev. Lett. 79, 2204 (1997)

[6] For recent developments, see the presentation by G. Giudice, this meeting.

[7] Presentations by Y. Suzuki, T. Mann, L. DiLella and H. Robertson, this meeting.

[8] N. Cabibbo, Phys. Rev. Lett. 10, 531 (1963).

[9] M. Kobayashi and T. Maskawa, Prog. Theor. Phys. 49, 652 (1973).

[10] L. Wolfenstein, Phys. Rev. Lett. 51, 1945 (1983).

[11] An excellent review is given by G. Buchalla, A.J. Buras and M.E. Lautenbacher, Rev. Mod. Phys. 68, 1125 (1996).

[12] S. Bosch et al., hep-ph/9904408.

[13] A review of chiral dynamics applied to this and other Standard Model processes may be found in J.F. Donoghue, E. Golowich and B.R. Holstein, Dynamics of the Standard Model, Cambridge University Press (1992).

[14] C. Caso et al. [Particle Data Group], Eur. Phys. J. C3, 1 (1998). 
[15] For a concise and well balanced review of heavy quark methods, applications and theoretical uncertainties, with extensive references to the original literature, see Z. Ligeti, presentation at KAON'99, Chicago, hep-ph/9908432.

[16] For a review of mixing and $\mathrm{CP}$ violation in $K$ and $B$ physics, including discussion of uncertainties and subleading corrections, see A.J. Buras and R. Fleischer, in Heavy Flavours II, eds. A.J. Buras and M. Lindner, World Scientific (1998).

[17] An excellent review of lattice QCD is given by S.R. Sharpe, hep-lat/9811006.

[18] Presentation by G. Blaylock, this meeting.

[19] P.F. Harrison and H.R. Quinn, eds. [BaBar Collaboration], The BaBar Physics Book: Physics at an Asymmetric B Factory, SLAC Report SLAC-R-504, 1998.

[20] F. Parodi, P. Roudeau and A. Stocchi, hep-ex/9903063.

[21] A.D. Sakharov, Zh. Eksp. Teor. Fiz. Pis'ma Red. 5, 32 [JETP Lett. 5, 24] (1967).

[22] A comprehensive review of electroweak baryogenesis is given by M. Trodden, hepph/9803479, to appear in Rev. Mod. Phys.; for a review of baryogenesis in the MSSM, see J.M. Cline, presentation at SEWM 98, Copenhagen, hep-ph/9902328.

[23] M. Gronau and D. London, Phys. Rev. Lett. 65, 3381 (1990).

[24] A.E. Snyder and H.R. Quinn, Phys. Rev. D48, 2139 (1993).

[25] See, for example, M. Neubert and J.L. Rosner, Phys. Rev. Lett. 81, 5076 (1998); R. Fleischer, hep-ph/9908341; R. Fleischer and T. Mannel, Phys. Rev. D57, 2752 (1998); M. Neubert, JHEP 02, 014 (1999).

[26] For recent efforts, see Y. Kwon et al. [CLEO Collaboration], CLEO CONF 99-14; M. Neubert and J.L. Rosner, Phys. Lett. B441, 403 (1998).

[27] D. Abbaneo et al. [LEP $V_{u b}$ Working Group], LEPVUB-99/01, 1999.

[28] B.H. Behrens et al. [CLEO Collaboration], hep-ex/9905056.

[29] Z. Ligeti and M.B. Wise, Phys. Rev. D53, 4937 (1996).

[30] C.G. Boyd, B. Grinstein and R.F. Lebed, Phys. Rev. Lett. 74, 4603 (1995); G. Burdman and J. Kambor, Phys. Rev. D55, 2817 (1997).

[31] A.F. Falk and A.A. Petrov, hep-ph/9903518; J. Chay, et al., hep-ph/9907363.

[32] Y. Grossman and M.P. Worah, Phys. Lett. B395, 241 (1997).

[33] Y. Grossman, Y. Nir and M.P. Worah, Phys. Lett. B407, 307 (1997). 


\section{Comments and Questions}

M. Peskin, SLAC: This morning, Ron Poling showed a fit for $\gamma$ from ratios of twobody exclusive $B$ branching ratios. [See also the CLEO preprint [26].] This result assumed that factorization is perfect, and so it has to be taken with a grain of salt. But do you think it is possible that such a determination of $\gamma$ could eventually be made "theoretically clean" in the sense that you used this term in your talk?

No, not in the restrictive sense in which I used the term. Analyses such as this one require severe assumptions about hadronic dynamics, assumptions which are then partially cross-checked within the analysis by examining the quality of the fit. But this ought not to be confused with actually understanding the physics of hadrons! The real test of an analysis is whether it is theoretically clean enough that one would be willing to rely on it for a definitive discovery of physics beyond the Standard Model. This is certainly not true of an analysis based on factorization. The natural explanation of any discrepancy with the Standard Model will be simply that the $a d$ hoc assumptions that had been made about hadronic dynamics were, in fact, wrong.

B. Ward, University of Tennessee: I would like to make a comment on the penguin pollution in $B \rightarrow \pi \pi$. There is an interplay between the phases as well as the size of the amplitude contributions (penguin and non-penguin), and in general there may be regions of parameter space in which $\sin 2 \alpha$ is measurable to some specificed accuracy even though the penguin amplitude's magnitude is large. Thus, I think your comments were a bit too pessimistic.

I disagree completely. It is true that hadronic physics may conspire to make the asymmetry in $B \rightarrow \pi \pi$ equal to $\sin 2 \alpha$ even in the presence of large penguin contributions. But this fact is of no use if the hadronic matrix elements, both magnitudes and phases, cannot be computed reliably from first principles. If $B \rightarrow \pi \pi$ is to provide any information on $V_{\mathrm{CKM}}$, we have to know unambiguously the relationship between the observed asymmetry and the operators of the LESM. The problem with penguin pollution is not simply that it may be large, it is that we do not know what it is!

K. Peach, RAL: Would you like to make a comment on when the lattice might be capable of making an accurate prediction for either $\epsilon$ or $\epsilon^{\prime}$ ?

No, I would not like to comment! I am not an expert on these issues, but I would say that it will be impossible to use lattice QCD to learn anything truly reliable until it is possible to go beyond the quenched approximation. This will start to come, hopefully, in the next generation of lattice computations. The interesting question is, at what point would the lattice people really be willing to claim that their calculations are airtight enough that they would rely on them for an unambiguous claim that new physics is required to explain the observed $\epsilon^{\prime}$ ? This will probably have to wait until unquenched calculations not only are available, but are themselves well understood. 